\title{
ON DECOMPOSABLE PSEUDOFREE GROUPS
}

\author{
DIRK SCEVENELS
}

(Received 16 October 1997)

\begin{abstract}
An Abelian group is pseudofree of rank $\ell$ if it belongs to the extended genus of $\mathbb{Z}^{\ell}$, i.e., its localization at every prime $p$ is isomorphic to $\mathbb{Z}_{p}^{\ell}$. A pseudofree group can be studied through a sequence of rational matrices, the so-called sequential representation. Here, we use these sequential representations to study the relation between the product of extended genera of free Abelian groups and the extended genus of their direct sum. In particular, using sequential representations, we give a new proof of a result by Baer, stating that two direct sum decompositions into rank one groups of a completely decomposable pseudofree Abelian group are necessarily equivalent. On the other hand, sequential representations can also be used to exhibit examples of pseudofree groups having nonequivalent direct sum decompositions into indecomposable groups. However, since this cannot occur when using the notion of near-isomorphism rather than isomorphism, we conclude our work by giving a characterization of near-isomorphism for pseudofree groups in terms of their sequential representations.
\end{abstract}

Keywords and phrases. Localization, extended genus, torsion-free Abelian group of finite rank, sequential representation, direct sum decomposition, near-isomorphism.

1991 Mathematics Subject Classification. 20K15, 20K25, 20F18.

1. Introduction. The Mislin genus $\mathscr{G}(N)[11]$ of a finitely generated nilpotent group $N$ is the set of isomorphism classes of finitely generated nilpotent groups $M$ such that the localization of $M$ and $N$ at every prime are isomorphic, that is $M_{p} \cong N_{p}$ for all primes $p$. If $N$ is a finitely generated Abelian group, then $N$ has a trivial Mislin genus. This observation led Casacuberta and Hilton [2] to introduce the notion of extended genus. They define the extended genus $\mathscr{E} \mathscr{G}(N)$ of a finitely generated nilpotent group $N$ as the set of isomorphism classes of (not necessarily finitely generated) nilpotent groups $M$ such that $M_{p} \cong N_{p}$ for all primes $p$. This notion admits interesting examples as, for example, in [6], Hilton showed that there are uncountably many nonisomorphic Abelian groups in the extended genus of $\mathbb{Z}$.

Following the definition of extended genus, the notion of a pseudofree group was introduced in [2, 6]. An Abelian group $A$ is called pseudofree of rank $\ell$-the name is due to Ries [12]-if $A$ belongs to the extended genus of $\mathbb{Z}^{\ell}$. Clearly, a pseudofree group of rank $\ell$ is torsion-free of rank $\ell$. However, the fact that $A$ is pseudofree imposes strong restrictions on $A$. This in turn enables us to use methods which do not seem to be applicable to the broad class of torsion-free Abelian groups of finite rank. In particular, we obtain the so-called "sequential representation" of a pseudofree group of rank $\ell$, which consists of a sequence of invertible $(\ell \times \ell)$-matrices (one for each prime) with entries in $\mathbb{Q}$.

For a finitely generated nilpotent group $N$ and for $k \geq 2$, the function 


$$
\Phi:(\mathscr{G}(N))^{k} \longrightarrow \mathscr{G}\left(N^{k}\right)
$$

sending the $k$-tuple $\left(L_{1}, \ldots, L_{k}\right)$ to $L_{1} \times \cdots \times L_{k}$ has been studied in $[3,7,8]$. If $N$ has a finite commutator subgroup, then $\Phi$ is surjective, but not necessarily injective. More generally, for finitely generated nilpotent groups $N_{1}, \ldots, N_{k}(k \geq 2)$, one can consider an obvious function (cf. [7]),

$$
\Psi: \mathscr{G}\left(N_{1}\right) \times \cdots \times \mathscr{G}\left(N_{k}\right) \longrightarrow \mathscr{G}\left(N_{1} \times \cdots \times N_{k}\right) .
$$

If all the groups $N_{1}, \ldots, N_{k}$ belong to a certain subclass of the class of finitely generated nilpotent groups with finite commutator subgroup, then this function is again surjective, but not necessarily injective (cf. [13]).

In Section 3, we study functions analogous to (1.1) and (1.2), now defined on the extended genus. It then makes sense to consider these functions when the groups involved are finitely generated and Abelian. As pointed out in [2], in this case, we may, without loss of generality, restrict our attention to the functions

$$
\tilde{\Phi}:\left(\mathscr{E} \mathscr{G}\left(\mathbb{Z}^{\ell}\right)\right)^{k} \longrightarrow \mathscr{E} \mathscr{G}\left(\mathbb{Z}^{\ell k}\right)
$$

and

$$
\tilde{\Psi}: \mathscr{E} \mathscr{G}\left(\mathbb{Z}^{\ell_{1}}\right) \times \cdots \times \mathscr{E} \mathscr{G}\left(\mathbb{Z}^{\ell_{k}}\right) \longrightarrow \mathscr{E} \mathscr{G}\left(\mathbb{Z}^{\ell_{1}+\cdots+\ell_{k}}\right),
$$

where $k \geq 2$ and $\ell, \ell_{1}, \ldots, \ell_{k}$ are positive integers. Of course, neither of these functions is surjective since there exist pseudofree Abelian groups that are not decomposable. If $\ell=1$, then we show that the function $\tilde{\Phi}$ is injective up to a reordering of the factors (see Corollary 3.2). In fact, this is a consequence of a well-known result due to Baer, stating that two direct sum decompositions into rank one groups of a completely decomposable Abelian torsion-free group of finite rank are necessarily equivalent (cf. [4, Thm. 86.1], [5, Thm. 117]). Using sequential representations, we give in Theorem 3.1 a new proof of Baer's result for completely decomposable pseudofree groups of finite rank. A proof for the case $k=2$ can also be found in [9]. On the other hand, it is well known that torsion-free Abelian groups of finite rank may have nonequivalent direct sum decompositions into indecomposable groups (cf. [4]). This implies that, if $\ell=2$, then $\tilde{\Phi}$ is far from being injective. Indeed, in Example 2.3, we use sequential representations to exhibit a pseudofree Abelian group $A$ of rank 4 with nonequivalent direct sum decompositions into indecomposable groups, i.e., $A \cong A_{1} \oplus A_{2} \cong B_{1} \oplus B_{2}$, where $A_{1} \cong A_{2}$ and $B_{1} \cong B_{2}$ are indecomposable pseudofree groups of rank2, but $A_{1} \npreceq B_{1}$. As to the function $\tilde{\Psi}$, in Example 2.4, we use sequential representations to give a pseudofree Abelian group $G$ of rank3 such that $G \cong A \oplus B \cong C \oplus D$, where $A \cong C$ are pseudofree groups of rank 1 and $B$ and $D$ are nonisomorphic pseudofree groups of rank2. In the course of Section 3, we thus provide an answer to various questions raised by Militello in [10].

Two torsion-free Abelian groups $A$ and $B$ of finite rank are called nearly isomorphic if, for every positive integer $n$, there is a subgroup $A_{n}$ of $B$, of finite index prime to $n$ such that $A_{n} \cong A$. Clearly, isomorphism implies near-isomorphism. The above mentioned phenomena of nonequivalent direct sum decompositions, however, 
cannot appear if one uses the notion of near-isomorphism rather than isomorphism. Indeed for torsion-free Abelian groups $A, B$, and $C$ of finite rank, if $A \oplus C$ is nearly isomorphic to $B \oplus C$, then $A$ is nearly isomorphic to $B$. Likewise, if $A^{k}$ is nearly isomorphic to $B^{k}$, then $A$ is nearly isomorphic to $B$ (cf. [1, Cor. 7.17]). In Section 4 , we therefore characterize nearly isomorphic pseudofree groups in terms of their sequential representations.

2. Pseudofree groups. For a finitely generated nilpotent group $N$, the extended genus $\mathscr{E} \mathscr{G}(N)$ [2] is the set of isomorphism classes of nilpotent groups $M$ such that $M_{p} \cong N_{p}$ for all primes $p$. The Mislin genus $\mathscr{G}(N)$ [11] is the subset of $\mathscr{E} \mathscr{G}(N)$ containing the isomorphism classes of finitely generated nilpotent groups. For short, we say that a group $M$ belongs to the extended (or Mislin) genus of $N$ if the isomorphism class of $M$ does.

For a finitely generated Abelian group $A$, the Mislin genus $\mathscr{G}(A)$ is easily seen to be trivial. However, the situation for $\mathscr{E} \mathscr{G}(A)$ is completely different. For example, the extended genus of $\mathbb{Z}$ contains uncountably many isomorphism classes (cf. [6]). In fact, in [2], Casacuberta and Hilton showed that in order to study the extended genus of any finitely generated Abelian group, it suffices to consider $\mathscr{E} \mathscr{G}\left(\mathbb{Z}^{\ell}\right)$. An Abelian group $A$ is called pseudofree of rank $\ell$ [12] if $A$ belongs to $\mathscr{E} \mathscr{G}\left(\mathbb{Z}^{\ell}\right)$. Note that if $A$ is pseudofree of rank $\ell$, then $A$ is torsion-free Abelian of rank $\ell$. For convenience of the reader, we recall from [2] the following basic facts about pseudofree groups. For a pseudofree group $A$ of rank $\ell$, we can choose isomorphism $f_{0}: A_{0} \cong \mathbb{Q}^{\ell}$ and $f_{p}: A_{p} \cong \mathbb{Z}_{p}^{\ell}$ for all primes $p$, which we write for short as $\left\{f_{p}, p \geq 0\right\}$. Since $A_{p}$ is naturally embedded in $A_{0}$, the homomorphism $f_{0} f_{p}^{-1}: \mathbb{Z}_{p}^{\ell} \rightarrow \mathbb{Q}^{\ell}$ is actually a monomorphism. With respect to the canonical basis $\left\{e_{1}, e_{2}, \ldots, e_{\ell}\right\}$ for $\mathbb{Z}^{\ell}, \mathbb{Z}_{p}^{\ell}, \mathbb{Q}^{\ell}$, this monomorphism $f_{0} f_{p}^{-1}$ is represented by a matrix $M_{p}$; explicitly,

$$
f_{0} f_{p}^{-1}\left(e_{j}\right)=\sum_{i} a_{i j}(p) e_{i}, \quad M_{p}=\left(a_{i j}(p)\right) \in \mathrm{GL}_{\ell}(\mathbb{Q})
$$

We write $M_{*}$ for the sequence of matrices $\left\{M_{p_{i}}\right\}$, where we enumerate the primes as $p_{1}, p_{2}, \ldots, p_{i}, \ldots$, and we call this sequence $M_{*}$ the sequential representation of $A$ associated with $\left\{f_{p}, p \geq 0\right\}$.

The pseudofree groups of rank one, the so-called groups of pseudo-integers, were treated by Hilton in [6]. For a group of pseudo-integers, a sequential representation simply consists of a sequence of rational numbers.

EXAMPLE 2.1. Let $A$ be a group of pseudo-integers. Then there exists a sequential representation $M_{*}$ of $A$ of the form $M_{p_{i}}=\left(p_{i}^{-m_{i}}\right)$, where $m_{i} \geq 0$. In fact, $A$ is isomorphic to the subgroup of $\mathbb{Q}$ generated by the set $\left\{p_{i}^{-m_{i}} \mid\right.$ all primes $\left.p_{i}\right\}$, and the sequence $\left(m_{i}\right)$ corresponds, in the classical sense, to a height sequence for a torsionfree Abelian group of rank one (cf. $[1,4,5])$.

A great deal of properties of pseudofree groups can be studied through their sequential representation. For future reference, we recall from [2] the following criterion for two pseudofree groups to be isomorphic. 
THEOREM 2.2. Let $A, B$ be pseudofree groups of rank $\ell$ with respective sequential representations $M_{*}$ and $N_{*}$. Then $A$ and $B$ are isomorphic if and only if

$$
\exists C_{p} \in \mathrm{GL}_{\ell}\left(\mathbb{Z}_{p}\right), L \in \mathrm{GL}_{\ell}(\mathbb{Q}): N_{p}=L M_{p} C_{p} \text { for all } p .
$$

In the case of groups of pseudo-integers, this leads to the following (cf. [6]).

EXAMPLE 2.3. Given groups of pseudo-integers $A, B$ with respective sequential representations $M_{*}$ and $N_{*}$ given by $M_{p_{i}}=\left(p_{i}^{-m_{i}}\right)$ and $N_{p_{i}}=\left(p_{i}^{-n_{i}}\right)$. Then $A$ is isomorphic to $B$ if and only if $m_{i}=n_{i}$ for almost all $i$. This corresponds with the fact that the isomorphism class of a torsion-free Abelian group of rank one is determined by its type (cf. [1, Thm. 1.1] and [4, Thm. 85.1]).

Particular attention will be paid to completely decomposable pseudofree groups. Recall that a torsion-free Abelian group $A$ of rank $\ell$ is called completely decomposable if

$$
A=\bigoplus_{i=1}^{\ell} A_{i},
$$

where each $A_{i}$ is torsion-free of rank one. Clearly, if $A$ is a completely decomposable pseudofree group of rank $\ell$ as in (2.3), then each $A_{i}$ is a group of pseudo-integers. Recall further (cf. [2, Thm. 4.3]) that a pseudofree group $A$ of rank $\ell$ is completely decomposable if and only if $A$ admits a sequential representation $D_{*}$, where each $D_{p}$ is a diagonal matrix.

3. Decomposable pseudofree groups. Let $N$ be a finitely generated nilpotent group with a finite commutator subgroup. From [7], we know that the Mislin genus $\mathscr{G}(N)$ has a natural Abelian group structure and that the function

$$
\Phi:(\mathscr{G}(N))^{k} \longrightarrow \mathscr{G}\left(N^{k}\right) \quad(\text { fork } \geq 2)
$$

given by

$$
\Phi\left(L_{1}, \ldots, L_{k}\right)=L_{1} \times \cdots \times L_{k}
$$

is actually a group homomorphism. In fact, $\Phi$ is a surjective homomorphism. This can be seen as follows. Recall from [3] that the function

$$
\rho: \mathscr{G}(N) \longrightarrow \mathscr{G}\left(N^{k}\right)
$$

given by

$$
\rho(L)=L \times N^{k-1}
$$

is a surjective homomorphism. Moreover, as explained in [7],

$$
\Phi\left(L_{1}, \ldots, L_{k}\right)=L_{1} \times \cdots \times L_{k} \cong\left(L_{1}+\cdots+L_{k}\right) \times N^{k-1}=\rho\left(L_{1}+\cdots+L_{k}\right),
$$

where + denotes the operation in the Abelian group $\mathscr{G}(N)$. Hence, since $\rho$ is surjective, we infer that $\Phi$ is surjective. In other words, every group in $\mathscr{G}\left(N^{k}\right)$ is decomposable.

On the other hand, it was pointed out in [7] that $\Phi$ is far from being injective. Indeed, in [7, Thm. 3.2], it is proved that, for $M_{i}, L_{i} \in \mathscr{G}(N)(i=1, \ldots, k)$, if $M_{1}+\cdots+M_{k}=$ $L_{1}+\cdots+L_{k}$ in $\mathscr{G}(N)$, then $M_{1} \times \cdots \times M_{k} \cong L_{1} \times \cdots \times L_{k}$. Moreover, in [8], the kernel of 
$\Phi$ is precisely described in the case where $N$ belongs to a certain subclass of the class of finitely generated nilpotent groups with finite commutator subgroup.

We now confine our attention to the analogue of the function $\Phi$, defined on the extended genus rather than the Mislin genus. As pointed out in [2], in order to study this function in the case when the groups involved are finitely generated Abelian, it suffices to consider the case of $\mathbb{Z}^{\ell}$. Thus, for $\ell \geq 1$ and $k \geq 2$, we consider the function

$$
\tilde{\Phi}:\left(\mathscr{E} \mathscr{G}\left(\mathbb{Z}^{\ell}\right)\right)^{k} \longrightarrow \mathscr{E} \mathscr{G}\left(\mathbb{Z}^{\ell k}\right)
$$

given by

$$
\tilde{\Phi}\left(L_{1}, \ldots, L_{k}\right)=L_{1} \times \cdots \times L_{k}
$$

Consider the case where $\ell=1$. It was pointed out in [2] that $\tilde{\Phi}: \mathscr{E} \mathscr{G}(\mathbb{Z})^{k} \rightarrow \mathscr{E} \mathscr{G}\left(\mathbb{Z}^{k}\right)$ is not surjective. Indeed, the image of $\tilde{\Phi}$ consists precisely of those pseudofree Abelian groups of rank $k$ that are completely decomposable. As to the injectivity of $\tilde{\Phi}$, a classical result by Baer (cf. [4, Prop. 86.1], [5, Thm. 117]) states that two direct sum decompositions into rank one groups of a completely decomposable torsion-free Abelian group of finite rank are necessarily equivalent. This means that if $A$ is a completely decomposable Abelian torsion-free group of rank $k$ and

$$
A \cong \bigoplus_{i=1}^{k} A_{i} \cong \bigoplus_{i=1}^{k} B_{i},
$$

where $A_{i}, B_{i}(i=1, \ldots, k)$ are torsion-free of rank one, then there exists a permutation $\sigma \in \Sigma_{k}$ such that $A_{i} \cong B_{\sigma(i)}$ for all $i$. Using sequential representations, we here give a new proof of this fact for pseudofree groups.

THEOREM 3.1. Let A be a completely decomposable pseudofree group of rank $k$. Suppose that

$$
A \cong \bigoplus_{i=1}^{k} A_{i} \cong \bigoplus_{i=1}^{k} B_{i},
$$

where $A_{i}, B_{i}(i=1, \ldots, k)$ are groups of pseudo-integers. Then there exists a permutation $\sigma \in \Sigma_{k}$ such that $A_{i} \cong B_{\sigma(i)}$ for all $i \in\{1, \ldots, k\}$.

Proof. We may assume that $A_{i} \cong\left\langle 1 / p^{m_{i}(p)}\right.$, all $\left.p\right\rangle$ and $B_{i} \cong\left\langle 1 / p^{n_{i}(p)}\right.$, all $\left.p\right\rangle$ for $i=1, \ldots, k$. Then clearly $A$ has a sequential representation $M_{*}$ given by

$$
M_{p}=\left(\begin{array}{cccc}
\frac{1}{p^{m_{1}(p)}} & 0 & \cdots & 0 \\
0 & \frac{1}{p^{m_{2}(p)}} & \cdots & 0 \\
\vdots & & \ddots & \vdots \\
0 & 0 & & \frac{1}{p^{m_{k}(p)}}
\end{array}\right),
$$


but $A$ also has a sequential representation $N_{*}$ given by

$$
N_{p}=\left(\begin{array}{cccc}
\frac{1}{p^{n_{1}(p)}} & 0 & \cdots & 0 \\
0 & \frac{1}{p^{n_{2}(p)}} & \cdots & 0 \\
\vdots & & \ddots & \vdots \\
0 & 0 & & \frac{1}{p^{n_{k}(p)}}
\end{array}\right) .
$$

Theorem 2.2 then implies that there exist $L \in \mathrm{GL}_{k}(\mathbb{Q})$ and $C_{p} \in \mathrm{GL}_{k}\left(\mathbb{Z}_{p}\right)$ such that $N_{p}=L M_{p} C_{p}$ for all primes $p$. Equivalently, $L^{-1} N_{p}=M_{p} C_{p}$ for all $p$. If we put $L^{-1}=$ $\left(\ell_{i j}\right)$ and $C_{p}=\left(c_{i j}(p)\right)$, then this is equivalent to

$$
\frac{\ell_{j i}}{p^{n_{i}(p)}}=\frac{c_{j i}(p)}{p^{m_{j}(p)}}
$$

for all $i, j \in\{1, \ldots, k\}$ and for all primes $p$. Since $\operatorname{det} C_{p}$ is a unit in $\mathbb{Z}_{p}$ and since the entries of $C_{p}$ belong to $\mathbb{Z}_{p}$, we infer that there exists a permutation $\sigma \in \Sigma_{k}$ such that $c_{\sigma(i) i}(p)$ is a unit in $\mathbb{Z}_{p}$ for all $i$. Moreover, since, for almost all $p$, the nonzero $\ell_{j i}$ are units in $\mathbb{Z}_{p}$, we conclude from (3.12) that

$$
m_{\sigma(i)}(p)=n_{i}(p)
$$

for almost all $p$. This implies that

$$
A_{i} \cong B_{\sigma(i)} \quad \text { for } i \in\{1, \ldots, k\} .
$$

COROLlarY 3.2. Let $\tilde{\Phi}$ be as in (3.6) with $\ell=1$. Then

$$
\tilde{\Phi}\left(L_{1}, \ldots, L_{k}\right)=\tilde{\Phi}\left(M_{1}, \ldots, M_{k}\right)
$$

if and only if $\left(L_{1}, \ldots, L_{k}\right)$ and $\left(M_{1}, \ldots, M_{k}\right)$ belong to the same orbit under the obvious action of $\Sigma_{k}$ on $(\mathscr{E} \mathscr{G}(\mathbb{Z}))^{k}$.

However, as the following example shows (cf. [1, Ex. 2.11]), the situation is completely different if we consider $\tilde{\Phi}$ defined on the extended genus of $\mathbb{Z}^{2}$.

EXAMPLE 3.3. Let $P_{1} \cup P_{2} \cup\{5\}$ be a partition of the set of all primes, where $P_{1}$ and $P_{2}$ are infinite. Consider the pseudofree group $A$ of rank 4 given by the sequential representation

$$
M_{p}=\left(\begin{array}{cccc}
\frac{1}{p_{1}} & 0 & 0 & 0 \\
0 & 1 & 0 & 0 \\
0 & 0 & \frac{1}{p_{1}} & 0 \\
0 & 0 & 0 & 1
\end{array}\right),\left(\begin{array}{cccc}
1 & 0 & 0 & 0 \\
0 & \frac{1}{p_{2}} & 0 & 0 \\
0 & 0 & 1 & 0 \\
0 & 0 & 0 & \frac{1}{p_{2}}
\end{array}\right),\left(\begin{array}{cccc}
1 & \frac{1}{5} & 0 & 0 \\
0 & \frac{1}{5} & 0 & 0 \\
0 & 0 & 1 & \frac{1}{5} \\
0 & 0 & 0 & \frac{1}{5}
\end{array}\right)
$$

for respectively $p=p_{1} \in P_{1}, p=p_{2} \in P_{2}$, and $p=5$. Consider also the pseudofree 
group $B$ of rank 4 given by the sequential representation

$$
N_{p}=\left(\begin{array}{cccc}
\frac{1}{p_{1}} & 0 & 0 & 0 \\
0 & 1 & 0 & 0 \\
0 & 0 & \frac{1}{p_{1}} & 0 \\
0 & 0 & 0 & 1
\end{array}\right),\left(\begin{array}{cccc}
1 & 0 & 0 & 0 \\
0 & \frac{1}{p_{2}} & 0 & 0 \\
0 & 0 & 1 & 0 \\
0 & 0 & 0 & \frac{1}{p_{2}}
\end{array}\right),\left(\begin{array}{cccc}
1 & \frac{1}{5} & 0 & 0 \\
0 & \frac{2}{5} & 0 & 0 \\
0 & 0 & 1 & \frac{1}{5} \\
0 & 0 & 0 & \frac{2}{5}
\end{array}\right)
$$

for respectively $p=p_{1} \in P_{1}, p=p_{2} \in P_{2}$, and $p=5$. Then clearly $A \cong A_{1} \oplus A_{2}$, where $A_{1} \cong A_{2}$ is an indecomposable pseudofree group of rank 2 with sequential representation

$$
X_{p}=\left(\begin{array}{cc}
\frac{1}{p_{1}} & 0 \\
0 & 1
\end{array}\right),\left(\begin{array}{ll}
1 & 0 \\
0 & \frac{1}{p_{2}}
\end{array}\right),\left(\begin{array}{ll}
1 & \frac{1}{5} \\
0 & \frac{1}{5}
\end{array}\right)
$$

for respectively $p=p_{1} \in P_{1}, p=p_{2} \in P_{2}$, and $p=5$. Analogously, $B \cong B_{1} \oplus B_{2}$, where $B_{1} \cong B_{2}$ is an indecomposable pseudofree group of rank 2 with sequential representation

$$
Y_{p}=\left(\begin{array}{cc}
\frac{1}{p_{1}} & 0 \\
0 & 1
\end{array}\right),\left(\begin{array}{ll}
1 & 0 \\
0 & \frac{1}{p_{2}}
\end{array}\right),\left(\begin{array}{ll}
1 & \frac{1}{5} \\
0 & \frac{2}{5}
\end{array}\right)
$$

for respectively $p=p_{1} \in P_{1}, p=p_{2} \in P_{2}$, and $p=5$. Observe that $A \cong B$. Indeed, setting

$$
L=\left(\begin{array}{cccc}
1 & 0 & 3 & 0 \\
0 & 2 & 0 & 1 \\
0 & 0 & -1 & 0 \\
0 & -5 & 0 & -2
\end{array}\right)
$$

and $C_{p}=L^{-1}$ for all $p \neq 5$, while

$$
C_{5}=\left(\begin{array}{cccc}
1 & 1 & 3 & 1 \\
0 & -4 & 0 & -2 \\
0 & -2 & -1 & -1 \\
0 & 10 & 0 & 4,
\end{array}\right)
$$

it is easily verified that $N_{p}=L M_{p} C_{p}$ for all primes $p$. On the other hand, note that $A_{1} \nRightarrow B_{1}$. Indeed, suppose that there exist $L \in \mathrm{GL}_{2}(\mathbb{Q})$ and $C_{p} \in \mathrm{GL}_{2}\left(\mathbb{Z}_{p}\right)$ such that $L X_{p} C_{p}=Y_{p}$ for all primes $p$. If we set

$$
L=\left(\begin{array}{ll}
x & z \\
y & t
\end{array}\right)
$$


then we infer that $C_{p}^{-1}=Y_{p}^{-1} L X_{p}$ is equal to

$$
\left(\begin{array}{cc}
x & p_{1} z \\
\frac{y}{p_{1}} & t
\end{array}\right),\left(\begin{array}{cc}
x & \frac{z}{p_{2}} \\
p_{2} y & t
\end{array}\right),\left(\begin{array}{cc}
x-\frac{1}{2} y & \frac{x+z}{5}-\frac{y+t}{10} \\
\frac{5}{2} y & \frac{y+t}{2}
\end{array}\right)
$$

for respectively $p=p_{1} \in P_{1}, p=p_{2} \in P_{2}$, and $p=5$. This implies that $p_{1} \mid y$ and $p_{2} \mid z$ for all $p_{1} \in P_{1}$ and for all $p_{2} \in P_{2}$. Hence, $y=0=z$. This means that

$$
C_{5}^{-1}=\left(\begin{array}{cc}
x & \frac{x}{5}-\frac{t}{10} \\
0 & \frac{t}{2}
\end{array}\right) .
$$

Moreover, since, for all $p$ different from 5, we have that

$$
C_{p}^{-1}=\left(\begin{array}{cc}
x & 0 \\
0 & t
\end{array}\right),
$$

we infer that $x=5^{i}$ and $t=5^{j}$ for some integers $i, j$. However, this is in contradiction with $C_{5}^{-1} \in \mathrm{GL}_{2}\left(\mathbb{Z}_{5}\right)$.

Another function of interest considered in [7] is the following. Let $N_{1}, \ldots, N_{k}$ be finitely generated nilpotent groups with a finite commutator subgroup and consider

$$
\Psi: \mathscr{G}\left(N_{1}\right) \times \cdots \times \mathscr{G}\left(N_{k}\right) \longrightarrow \mathscr{G}\left(N_{1} \times \cdots \times N_{k}\right)
$$

given by

$$
\Psi\left(L_{1}, \ldots, L_{k}\right)=L_{1} \times \cdots \times L_{k}
$$

In [7], it is shown that $\Psi$ is a group homomorphism and the authors asked whether $\Psi$ is always surjective. In [13], this homomorphism $\Psi$ is studied for a certain subclass of the class of finitely generated nilpotent groups with finite commutator subgroup. In this case, $\Psi$ is indeed surjective and, moreover, the exact conditions for $\Psi\left(L_{1}, \ldots, L_{k}\right)=$ $\Psi\left(M_{1}, \ldots, M_{k}\right)$ (i.e., for $\left.L_{1} \times \cdots \times L_{k} \cong M_{1} \times \cdots \times M_{k}\right)$ to hold are described.

Again, we consider the analogue function defined on the extended genus and we are particularly interested in

$$
\tilde{\Psi}: \mathscr{E} \mathscr{G}\left(\mathbb{Z}^{\ell_{1}}\right) \times \cdots \times \mathscr{E} \mathscr{G}\left(\mathbb{Z}^{\ell_{k}}\right) \longrightarrow \mathscr{E} \mathscr{G}\left(\mathbb{Z}^{\ell_{1}+\cdots+\ell_{k}}\right) .
$$

Of course, this function $\tilde{\Psi}$ is not surjective. Indeed, not every pseudofree group of finite rank is decomposable. Furthermore, $\tilde{\Psi}$ is far from being injective in general. Indeed, it is a well-known fact that torsion-free Abelian groups of finite rank may have nonequivalent direct sum decompositions (cf. [4, Thm. 90.4]), In fact, we can already exhibit a pseudofree group of rank 3 that has two nonequivalent direct sum decompositions (cf. [1, Ex. 2.10]).

EXAMPLE 3.4. Let $P_{1} \cup P_{2} \cup\{5\}$ be a partition of the set of all primes, where $P_{1}$ and $P_{2}$ are infinite. Consider the pseudofree group $G$ of rank 3 given by the sequential 
representation

$$
M_{p}=\left(\begin{array}{ccc}
\frac{1}{p_{1}} & 0 & 0 \\
0 & \frac{1}{p_{1}} & 0 \\
0 & 0 & 1
\end{array}\right),\left(\begin{array}{ccc}
1 & 0 & 0 \\
0 & 1 & 0 \\
0 & 0 & \frac{1}{p_{2}}
\end{array}\right),\left(\begin{array}{ccc}
1 & 0 & 0 \\
0 & 1 & \frac{1}{5} \\
0 & 0 & \frac{1}{5}
\end{array}\right)
$$

for respectively $p=p_{1} \in P_{1}, p=p_{2} \in P_{2}$, and $p=5$. Consider also the pseudofree group $H$ of rank 3 given by the sequential representation

$$
N_{p}=\left(\begin{array}{ccc}
\frac{8}{p_{1}} & \frac{5}{p_{1}} & 0 \\
\frac{3}{p_{1}} & \frac{2}{p_{1}} & 0 \\
0 & 0 & 1
\end{array}\right),\left(\begin{array}{ccc}
1 & 0 & 0 \\
0 & 1 & 0 \\
0 & 0 & \frac{1}{p_{2}}
\end{array}\right),\left(\begin{array}{ccc}
1 & 0 & 3 \\
0 & 1 & \frac{6}{5} \\
0 & 0 & \frac{1}{5}
\end{array}\right)
$$

for respectively $p=p_{1} \in P_{1}, p=p_{2} \in P_{2}$, and $p=5$. Then it is easily seen that $G \cong$ $A \oplus B$, where $A$ is pseudofree of rank 1 with sequential representation ( $\left.1 / p_{1}\right),(1),(1)$ for respectively $p=p_{1} \in P_{1}, p=p_{2} \in P_{2}$, and $p=5$, and $B$ is pseudofree of rank 2 with sequential representation

$$
\left(\begin{array}{cc}
\frac{1}{p_{1}} & 0 \\
0 & 1
\end{array}\right),\left(\begin{array}{cc}
1 & 0 \\
0 & \frac{1}{p_{2}}
\end{array}\right),\left(\begin{array}{ll}
1 & \frac{1}{5} \\
0 & \frac{1}{5}
\end{array}\right)
$$

for respectively $p=p_{1} \in P_{1}, p=p_{2} \in P_{2}$, and $p=5$. Analogously, it can be verified that $H \cong C \oplus D$, where $C$ is pseudofree of rank 1 with sequential representation $\left(1 / p_{1}\right),(1),(1)$ for respectively $p=p_{1} \in P_{1}, p=p_{2} \in P_{2}$, and $p=5$, and $D$ is pseudofree of rank 2 with sequential representation

$$
\left(\begin{array}{cc}
\frac{1}{p_{1}} & 0 \\
0 & 1
\end{array}\right),\left(\begin{array}{cc}
1 & 0 \\
0 & \frac{1}{p_{2}}
\end{array}\right),\left(\begin{array}{ll}
1 & \frac{3}{5} \\
0 & \frac{1}{5}
\end{array}\right)
$$

for respectively $p=p_{1} \in P_{1}, p=p_{2} \in P_{2}$, and $p=5$. Finally, observe that $G \cong H$ and $A \cong C$ while $B \nsubseteq D$.

4. Near-isomorphism of pseudofree groups. Motivated by the fact that the above phenomena of nonequivalent direct sum decompositions of a pseudofree group cannot appear if we use the notion of near-isomorphism rather than the notion of isomorphism (cf. [1, Cor. 7.17]), we give in this section a characterization of near-isomorphism for pseudofree groups in terms of their sequential representations.

Recall (see, e.g., [1]) that two torsion-free Abelian groups $A$ and $B$ of finite rank are called nearly isomorphic (notation $A \cong_{n} B$ ) if, for every positive integer $n$, there is a subgroup $A_{n}$ of $B$, of finite index prime to $n$ such that $A_{n} \cong A$. Note that $A$ and $B$ are then necessarily of the same rank. Moreover, two nearly isomorphic torsion-free Abelian groups obviously belong to the same extended genus.

THEOREM 4.1. Let $A$ and $B$ be pseudofree groups of rank $k$ with respectively sequential representations $M_{*}$ and $N_{*}$, induced by $\left\{f_{p}, p \geq 0\right\}$ and $\left\{f_{p}^{\prime}, p \geq 0\right\}$. Then $A \cong_{n} B$ 
if and only if, for all positive integers $n$, there exists $\Phi_{n} \in \mathrm{GL}_{k}(\mathbb{Q})$ such that $N_{p}^{-1} \Phi_{n} M_{p}$ has entries in $\mathbb{Z}_{p}$ for all primes $p$, and $N_{p}^{-1} \Phi_{n} M_{p} \in \mathrm{GL}_{k}\left(\mathbb{Z}_{p}\right)$ for almost all primes $p$, including all the prime divisors of $n$.

Proof. Suppose that $A \cong_{n} B$. For all positive integers $n$, there exists an embedding $j_{n}: A \rightarrow B$ of finite index such that $\left[B: j_{n}(A)\right]=k_{n}$ is prime to $n$. Then $\left(j_{n}\right)_{0}: A_{0} \rightarrow B_{o}$ is an isomorphism and we may choose $\left(f_{n}\right)_{0}: A_{0} \rightarrow \mathbb{Q}^{k},\left(f_{n}^{\prime}\right)_{0}: B_{0} \rightarrow \mathbb{Q}^{k}$ such that the following diagram commutes.

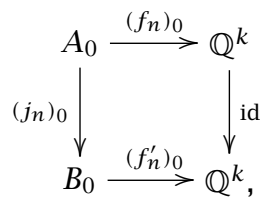

i.e., $\left(f_{n}\right)_{0}=\left(f_{n}^{\prime}\right)_{0} \circ\left(j_{n}\right)_{0}$. This means that we change the sequential representations $M_{*}$ and $N_{*}$ to respectively $\tilde{M}_{*}, \tilde{N}_{*}$ given by

$$
\tilde{M}_{p}=S_{n} M_{p}, \quad \tilde{N}_{p}=S_{n}^{\prime} N_{p},
$$

where $S_{n}, S_{n}^{\prime} \in \mathrm{GL}_{k}(\mathbb{Q})$. Since now the conditions of [2, Thm. 2.6] are satisfied, we infer that

$$
\left(\tilde{N}_{p}\right)^{-1} \tilde{M}_{p}
$$

has entries in $\mathbb{Z}_{p}$ for all primes $p$. This is equivalent to

$$
N_{p}^{-1} \Phi_{n} M_{p}
$$

having entries in $\mathbb{Z}_{p}$ for all $p$, where $\Phi_{n}=S_{n}^{\prime-1} S_{n}$. Moreover, since $\left[B: j_{n}(A)\right]=k_{n}$ is prime to $n$, we know that $\left(j_{n}\right)_{p}: A_{p} \rightarrow B_{p}$ is an isomorphism for all primes $p$ not dividing $k_{n}$. If $p$ does not divide $k_{n}$, then we have the following diagram:

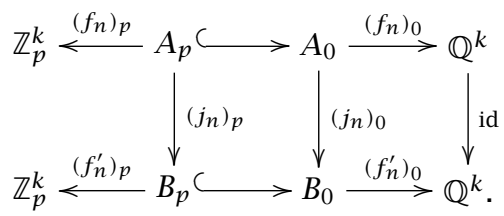

Hence, if $p$ does not divide $k_{n}$, then there exists an isomorphism $\gamma_{n}(p): \mathbb{Z}_{p}^{k} \rightarrow \mathbb{Z}_{p}^{k}$ such that $\left(f_{n}^{\prime}\right)_{0} \circ\left(f_{n}^{\prime}\right)_{p}^{-1} \circ \gamma_{n}(p)=\left(f_{n}\right)_{0} \circ\left(f_{n}\right)_{p}^{-1}$. This means that the matrix of $\gamma_{n}(p)$, given by

$$
\left(S_{n}^{\prime} N_{p}\right)^{-1} S_{n} M_{p}=N_{p}^{-1} \Phi_{n} M_{p}
$$

belongs to $\mathrm{GL}_{k}\left(\mathbb{Z}_{p}\right)$ if $p \nmid k_{n}$.

Conversely, let the isomorphisms $\phi_{n}: \mathbb{Q}^{k} \rightarrow \mathbb{Q}^{k}$ correspond to the given matrices $\Phi_{n}$. Then the composition

$$
\mathbb{Z}_{p}^{k} \stackrel{f_{p}^{-1}}{\longrightarrow} A_{p} \longrightarrow A_{0} \stackrel{f_{0}}{\longrightarrow} \mathbb{Q}^{k} \stackrel{\phi_{n}}{\longrightarrow} \mathbb{Q}^{k}
$$

corresponds to a sequential representation for $A$, given by $\Phi_{n} M_{p}$, and induced by 
homomorphisms $\left(g_{n}\right)_{0}:=\phi_{n} \circ f_{0}$ and $\left(g_{n}\right)_{p}:=f_{p}$. By [2, Thm. 2.6], we find a homomorphism $j_{n}: A \rightarrow B$ such that $\left(g_{n}\right)_{0}=f_{0}^{\prime} \circ\left(j_{n}\right)_{0}$. Moreover, due to the hypothesis on $N_{p}^{-1} \Phi_{n} M_{p}$, we deduce the existence of a monomorphism $\gamma_{n}(p): \mathbb{Z}_{p}^{k} \rightarrow \mathbb{Z}_{p}^{k}$, which is an isomorphism for almost all primes $p$, including all prime divisors of $n$. Hence, it induces a monomorphism $j_{n}(p): A_{p} \rightarrow B_{p}$ (which is an isomorphism for almost all primes, including the prime divisors of $n$ ) such that the following diagram commutes.

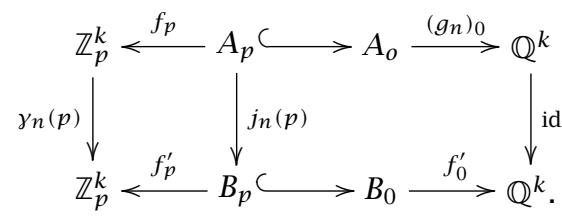

Now set $j_{n}(0)=f_{o}^{\prime-1} \circ\left(g_{n}\right)_{0}$. Then the diagram

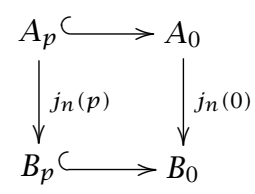

commutes for all primes $p$. Hence, there exists a monomorphism $j_{n}: A \rightarrow B$ such that $\left(j_{n}\right)_{p}=j_{n}(p)$, which is an isomorphism for almost all $p$, including the prime divisors of $n$. Finally, it is easily verified that $j_{n}$ is, in fact, an embedding of finite index prime to $n$.

EXAMPLE 4.2. With the notations as in Example 2.3, we show that $A_{1} \cong_{n} B_{1}$. Let $n$ be any positive integer and suppose that its prime divisors are $p_{1}, \ldots, p_{k}$. Choose an integer $m$ such that $2+5 m$ is a prime number different from $p_{1}, \ldots, p_{k}$ and set

$$
\Phi_{n}=\left(\begin{array}{cc}
1 & 0 \\
0 & 2+5 m
\end{array}\right) .
$$

Then it is easy to verify that $Y_{p}^{-1} \Phi_{n} X_{p}$ has entries in $\mathbb{Z}_{p}$ for all primes $p$ and that it belongs to $\mathrm{GL}_{2}\left(\mathbb{Z}_{p}\right)$ for almost all primes, including $p_{1}, \ldots, p_{k}$.

\section{REFERENCES}

[1] D. M. Arnold, Finite Rank Torsion Free Abelian Groups and Rings, Lecture Notes in Mathematics, vol. 931, Springer-Verlag, Berlin, New York, 1982. MR 84d:20002. Zbl 493.20034.

[2] C. Casacuberta and P. Hilton, On the extended genus of finitely generated Abelian groups, Bull. Soc. Math. Belg. Sér. A 41 (1989), no. 1, 51-72. MR 91j:20089. Zbl 672.20024.

[3] Calculating the Mislin genus for a certain family of nilpotent groups, Comm. Algebra 19 (1991), no. 7, 2051-2069. MR 92j:20032. Zbl 742.20034.

[4] L. Fuchs, Infinite Abelian Groups. II, Pure and Applied Mathematics, vol. 36-II, Academic Press, New York, London, 1973. MR 50 2362. Zbl 257.20035.

[5] P. A. Griffith, Infinite Abelian Group Theory, Chicago Lectures in Mathematics, The University of Chicago Press, Chicago, 1970. MR 44\#6826. Zbl 204.35001.

[6] P. Hilton, On groups of pseudo-integers, Acta Math. Sinica (N.S.) 4 (1988), no. 2, 189-192. MR 89i:11015. Zbl 682.20039. 
[7] P. Hilton and G. Mislin, On the genus of a nilpotent group with finite commutator subgroup, Math. Z. 146 (1976), no. 3, 201-211. MR 53 615. Zbl 311.20013.

[8] P. Hilton, D. Scevenels, and C. Schuck, Non-cancellation phenomena for direct products in a class of finitely generated nilpotent groups, Preprint, 1996.

[9] R. Militello and H. Ries, On pseudofree groups and sequential representations, New Zealand J. Math. 23 (1994), no. 2, 137-146. MR 96c:20103. Zbl 826.20051.

[10] R. R. Militello, On a certain class of nilpotent groups, Group theory (River Edge, NJ) (S. Sehgal et al., eds.), World Sci. Publishing, 1993, pp. 229-236. MR 96f:20055. Zbl 831.20042.

[11] G. Mislin, Nilpotent groups with finite commutator subgroups, Localization in group theory and homotopy theory, and related topics (Berlin), Lecture Notes in Math., vol. 418, Springer, 1974, pp. 103-120. MR 50 10080. Zbl 302.20029.

[12] H. Ries, On torsion free Abelian groups that are almost finitly-generated, Ph.D. thesis, SUNY, Binghamton, 1992.

[13] D. Scevenels, The genus of a direct product of nilpotent groups of a certain type and non-cancellation phenomena, Comm. Algebra 23 (1995), no. 9, 3223-3231. MR 96c:20062. Zbl 843.20029.

SCEVenels: Centre de Recerca MatemÀtica, Apartat 50, E-08193 Bellaterra, Spain 


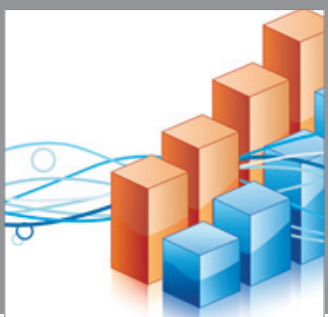

Advances in

Operations Research

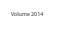

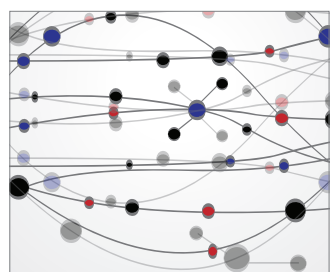

\section{The Scientific} World Journal
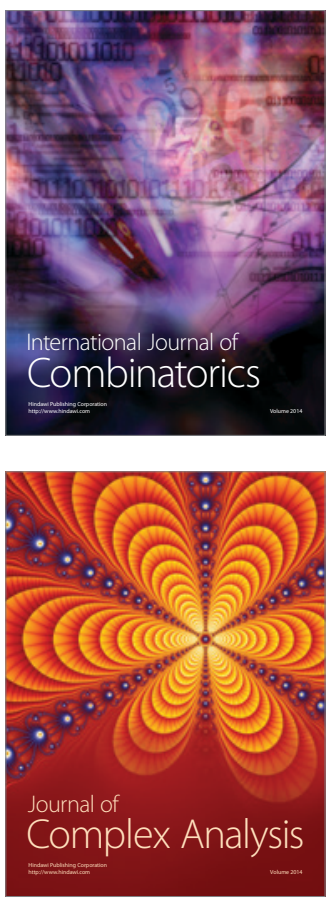

International Journal of

Mathematics and

Mathematical

Sciences
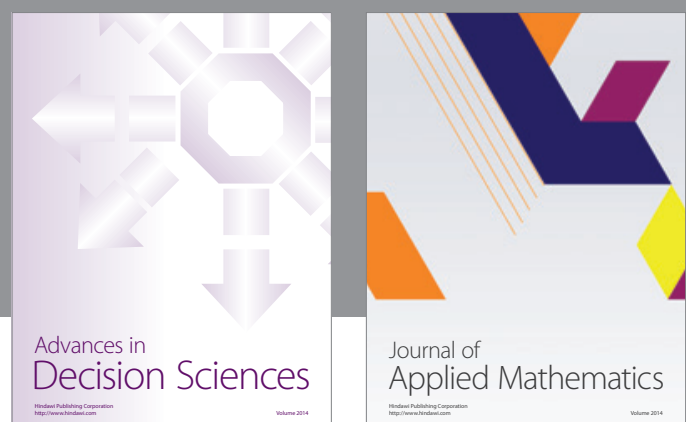

Journal of

Applied Mathematics
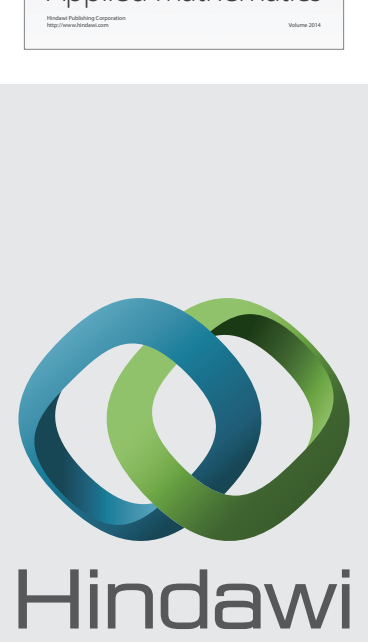

Submit your manuscripts at http://www.hindawi.com
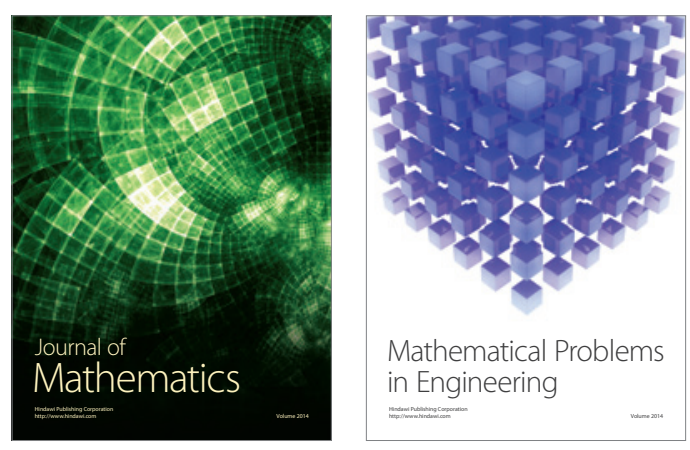

Mathematical Problems in Engineering
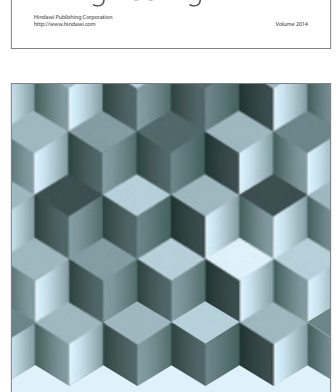

Journal of

Function Spaces
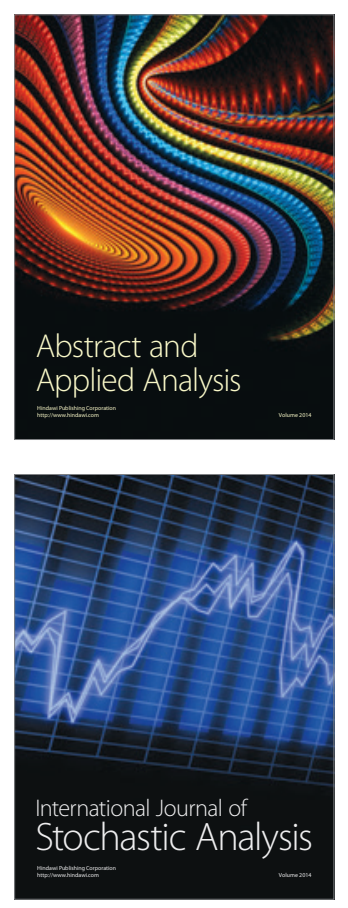

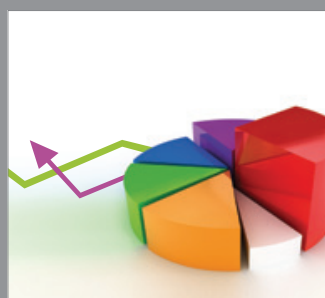

ournal of

Probability and Statistics

Promensencen
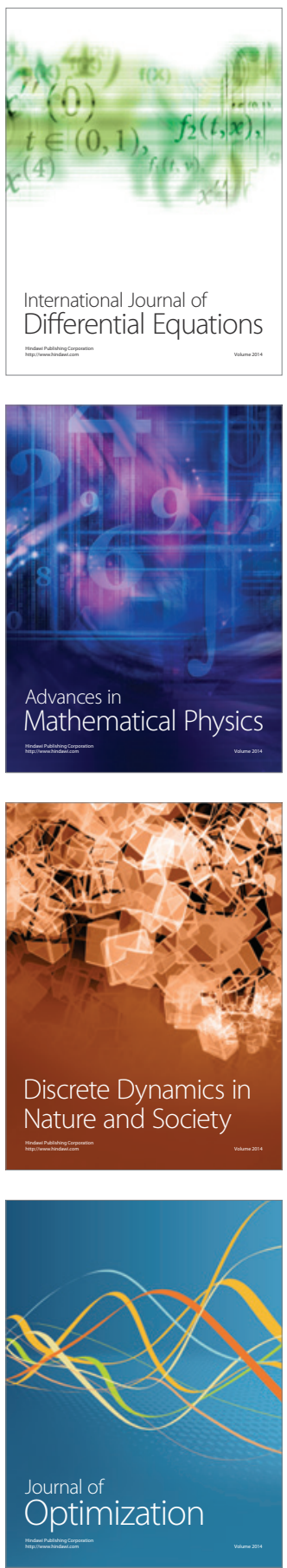